\title{
Simultaneous observation of two stars using the PRIMA Star Separator
}

\author{
J. Nijenhuis ${ }^{\mathrm{a}}$, H. Visser ${ }^{\mathrm{a}}$, H. de Man ${ }^{\mathrm{a}}$, B. Dekker ${ }^{\mathrm{a}}$, J. Mekking ${ }^{\mathrm{a}}$, F. Kamphues ${ }^{\mathrm{a}}$ \\ ${ }^{\mathrm{a}}$ TNO Science \& Industry, Stieltjesweg 1, 2628 CK, Delft, The Netherlands
}

\begin{abstract}
ESO is building the Phase Referenced Imaging and Microarcsecond Astronomy (PRIMA) facility for the Very Large Telescope Interferometer (VLTI) in Chile. PRIMA will enable interferometric imaging of very faint objects and high precision astrometry with both Unit (UT) and Auxiliary (AT) telescopes. The PRIMA facility consists of four major sub systems: Star Separators, Differential Delay Lines, Metrology and Fringe Sensor Units. TNO has developed the PRIMA Star Separator (STS) subsystems for both the UT and AT telescopes. The STS separates the light of two astronomical objects and feeds it into the long stroke delay line. The STS compensates for field rotation, stabilizes the beam tip tilt and adjust the lateral and axial alignment of the pupil. Chopping and/or counter-chopping on the science object or the guide star has also been implemented.
\end{abstract}

Keywords: European Southern Observatory, ESO, Very Large Telescope Interferometer, VLTI, PRIMA, star separator, STS, astrometry, interferometry, aperture synthesis, scan mechanism

\section{INTRODUCTION}

PRIMA will make use of the dual feed capability of the Very Large Telescope Interferometer for both Unit and Auxiliary Telescopes (Figure 1). With PRIMA the global limiting magnitude of the VLTI will gain about six magnitudes in $\mathrm{H}, \mathrm{K}$ and $\mathrm{N}$ bands. PRIMA will perform high accuracy astrometry (10 $\mu$ as) in band $\mathrm{H}$ or $\mathrm{K}$, aperture synthesis (phase reference imaging) and model constrained imaging in bands $\mathrm{J}, \mathrm{H}, \mathrm{K}$ and $\mathrm{N}$ [1]. Key science objectives are detection of Jupiter size exoplanets and exploration of nuclear regions of galaxies, including our own.

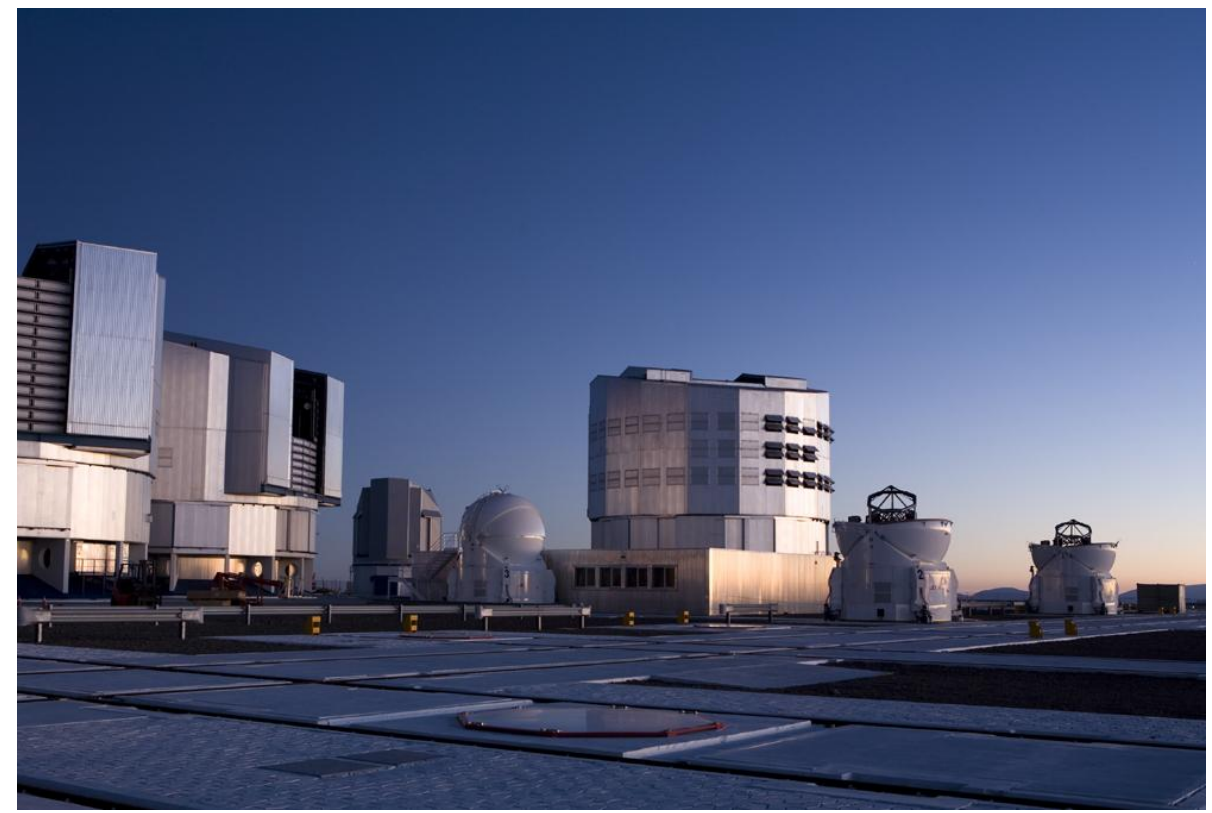

Figure 1: The Very Large Telescope Interferometer at Cerro Paranal (courtesy ESO)

Optical and Infrared Interferometry, edited by Markus Schöller, William C. Danchi, Françoise Delplancke Proc. of SPIE Vol. 7013, 70133F, (2008) · 0277-786X/08/\$18 · doi: 10.1117/12.789943 
ben.braam@tno.nl; phone +31(0)152692180; fax +31(0)152692111; www.tno.nl

PRIMA will be composed of four major sub-systems: Star Separator, Differential Delay Lines, Metrology and Fringe Sensor Units.

Interferometry on relatively bright stars improves the detectability of nearby faint objects (up to 120 " separation on sky). Objects that were first unobservable by interferometry due to the atmospheric turbulence now become visible. These faint objects become visible because:

- The bright star (guide star) is used to compensate the turbulence induced by the atmosphere (by making short exposures during which the atmospheric turbulence is "frozen" and by correcting for the measured delay).

- The turbulence is then also compensated in the direct environment of the star and the signal on the very faint object (called science star) is not "diluted" anymore, can be integrated longer and finally detected.

The function of the Star Separator is to enable simultaneous observation of two objects; one being the guide star and the other being the science object. The latter could be a Jupiter like planet or another very faint star.

TNO has developed the PRIMA Star Separator subsystems for both the UT and AT telescopes.

\section{REQUIREMENTS}

The STS separates the light of two astronomical objects and feeds it into the long stroke delay line. The STS compensates for field rotation, stabilizes the beam tip tilt and adjust the lateral and axial alignment of the pupil. Chopping and/or counter-chopping on the science object or the guide star has also been implemented.

The main requirements for the Star Separators are:

- Simultaneous observation of two objects in the Coude focal plane. The objects are located within 2 arcmin of each other.

- Pointing and tracking of stars. The Star Separator must compensate for the Earth's movement with an accuracy 0.01 arcsec and 0.002 arcsec resolution (for the UT STS only, for the AT STS this is realized with a separate de-rotator).

- Chopping on a star. Pointing at an object and its dark background with 1-5 Hz frequency provides the option to subtract the background from the object

- Tip-tilt control. The output should be controlled in tip-tilt to align it with the delay lines and subsequently the detectors.

- Pupil alignment. The output pupil must be carefully aligned with the input pupil, to avoid vignetting and to ensure a proper overlap for the metrology system.

\section{AT STAR SEPARATOR}

The AT telescopes are equipped with a de-rotator to compensate for the diurnal motion. The de-rotator and Star Separator are mounted in a Relay Optics Structure (ROS) underneath the AT (Figure 2).

Figure 3 shows the optical layout of the AT Star Separator. The Coude focus of the telescope coincides with the rooftop mirror M10 that separates the telescope Field of View into two sections. The de-rotator in front of the STS assures that the guide star and the science object are re-imaged at either side of the rooftop. M10 re-images the telescope pupil on both flat folding mirrors M11. Mirror M12 re-images the Coude focus on M14. Because the pupil coincides with M11, it is possible to point at any re-imaged object on M10 by a small rotation around two perpendicular axes of M11. 


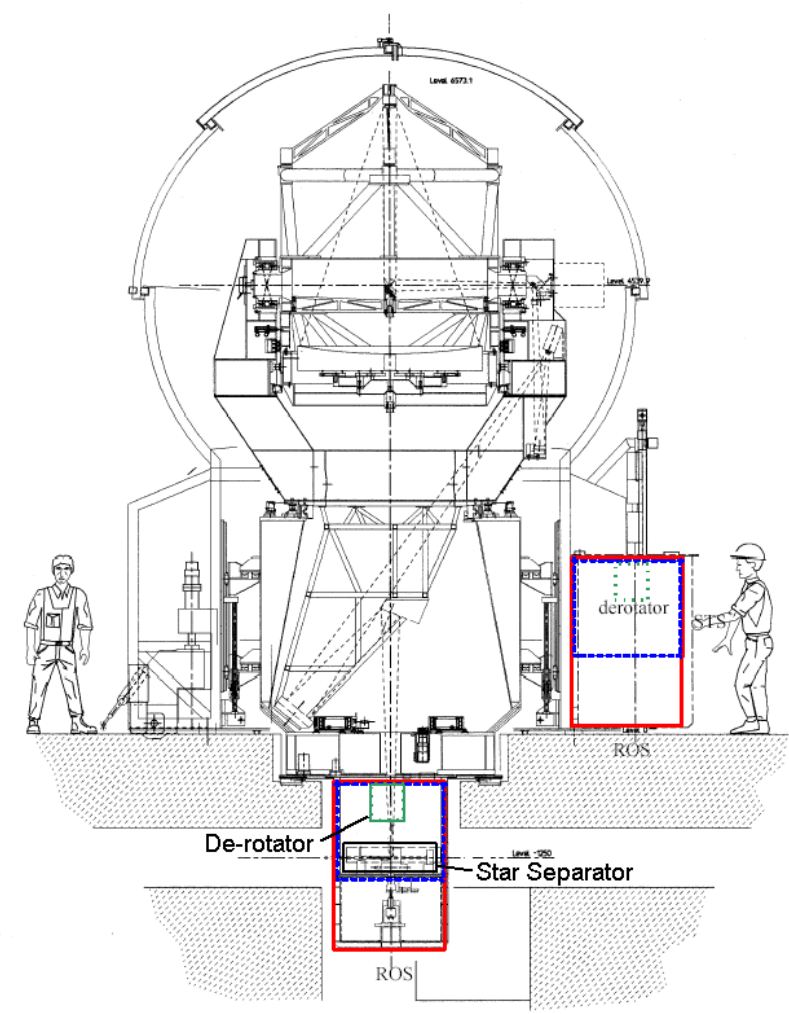

Figure 2: AT telescope with de-rotator and star separator

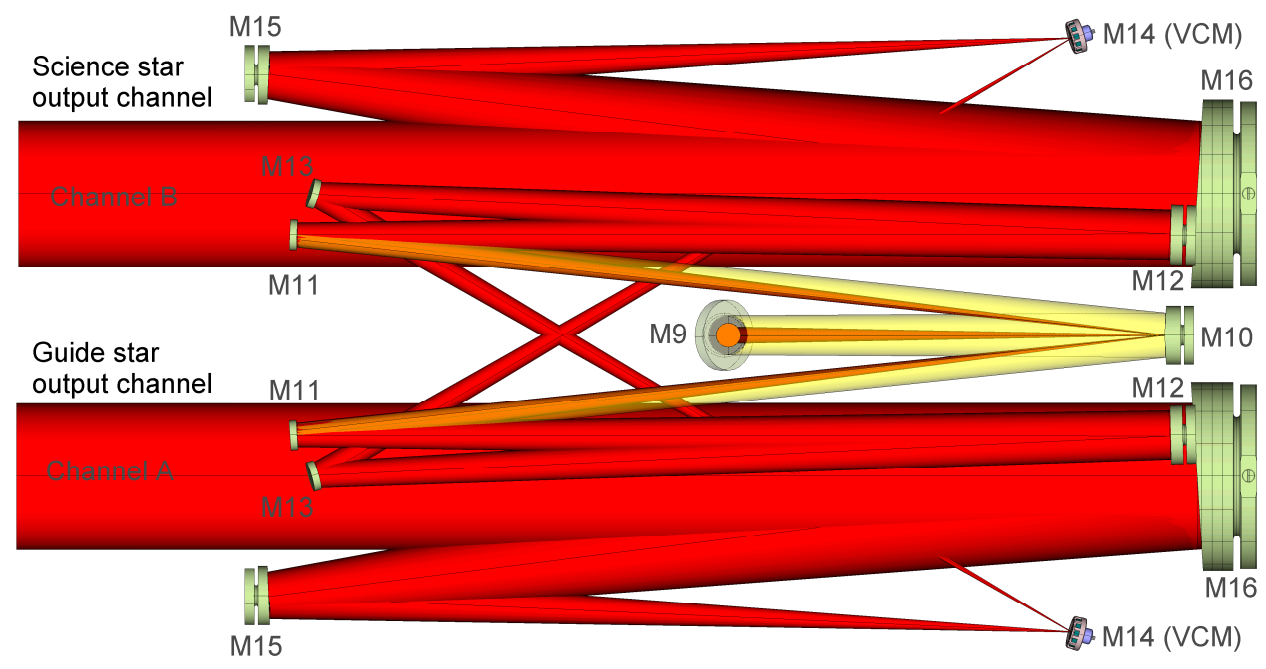

Figure 3: Optical layout of AT Star Separator

Proc. of SPIE Vol. 7013 70133F-3 
Stability of the STS is of utmost importance. Therefore temperature changes and mechanical vibrations should have negligible consequences. Furthermore friction inside the mechanisms is not allowed because of the resulting hysteresis. The size of M11 is only Ø25 mm and guarantees that the moving mass is low ( $\approx 40$ gram) and will not hamper the dynamics of the mechanism during chopping. By rotating M11 around two orthogonal axes it is possible to point at the object of interest that is re-imaged anywhere on M10. Table 1 specifies the requirements for the M11 mechanisms.

Table 1: M11 Mechanism requirements

\begin{tabular}{|l|l|l|l|}
\hline Requirement & Required value & Requirement & Required value \\
\hline Pointing range & $14.6 \mathrm{mrad}$ (both axes) $\approx 1^{\circ}$ & Pointing correction freq. & $50 \mathrm{~Hz}$ \\
\hline Pointing accuracy & $2.1 \mu \mathrm{rad}$ & Chopping frequency & $1 \mathrm{~Hz}(5 \mathrm{~Hz}$ goal $)$ \\
\hline Pointing resolution & $0.43 \mu \mathrm{rad}$ & Chopping accuracy & $94 \mu \mathrm{rad}$ \\
\hline
\end{tabular}

For the M11 mechanism two elements are indispensable. These two elements are:

1. The guiding mechanism for the pupil mirror.

2. The actuating mechanism that provides the necessary mirror rotation.

Based on the requirements it is clear that a solution based on flexures is the only feasible approach. This way hysteresis in the mechanical section of the mechanism can be avoided.

The mechanism consists of a monolithic guiding mechanism for the mirror and a separate actuation section. The guiding mechanism is shown in Figure 4. It consists of four folded leaf springs. The folded leaf springs constrain 4 degrees of freedom.

A cross section of the actuator mechanism is shown in Figure 5. The mechanism is actuated by two piezo stacks from PI. The piezo displacement is amplified by a monolithic lever mechanism. The piezo provides a maximum travel of 38 $\mu \mathrm{m}$ with a repeatability of $3 \mathrm{~nm}$ and a resolution of $2 \AA$. These performances are possible because of the capacitive sensor that is built into the piezo actuator. The equivalent mirror rotations are: $16 \mathrm{mrad}, 1.3 \mu \mathrm{rad}$ and $0.08 \mu \mathrm{rad}$. This is better than the required values as given in Table 1.

The M11 mechanisms are shown in Figure 6.

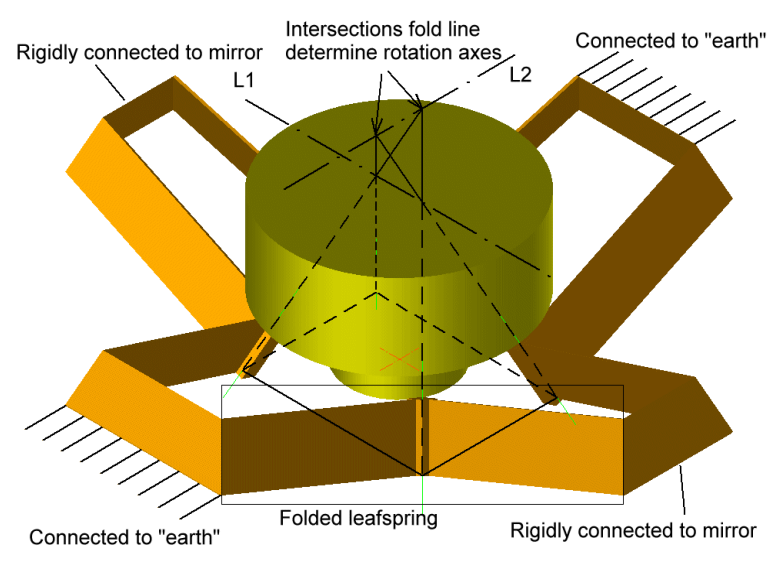

Figure 4: Schematic layout of guiding principle of M11 mechanism

The fully assembled AT Star Separator is shown in Figure 7. The AT star separators are mounted in a Relay Optics Structure (ROS) as shown in Figure 8.

To verify the performance of the mechanism it was mounted on a flat table with a fixed auto collimator in front of it. The resolution of this collimator is $0.1 \mu \mathrm{rad}$ with an accuracy of $0.5 \mu \mathrm{rad}$. Repeatability is just as good as the auto 
collimator could measure it i.e. it is $0.5 \mu \mathrm{rad}$. The chopping features of the mechanism were tested as well. Chopping at $5 \mathrm{~Hz}$ proved to be no problem. Even faster actuation is possible. For pointing correction during observations ESO would like to correct at $50 \mathrm{~Hz}$. The limit of the mechanism is $>100 \mathrm{~Hz}$.

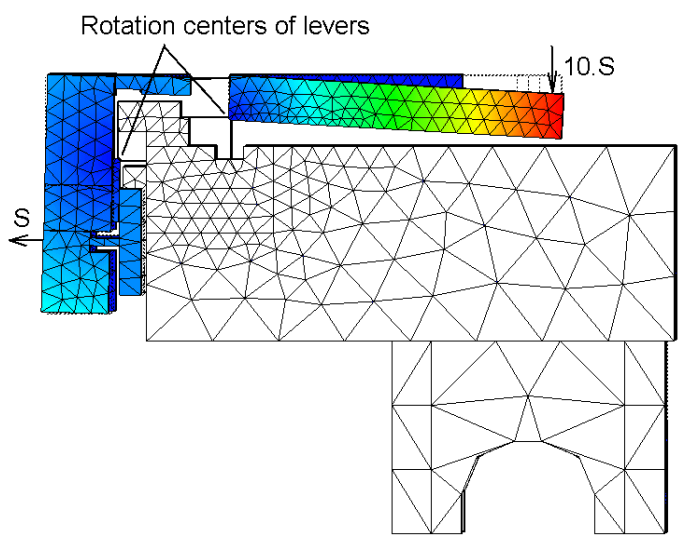

Figure 5: Horizontal cross section of actuator mechanism

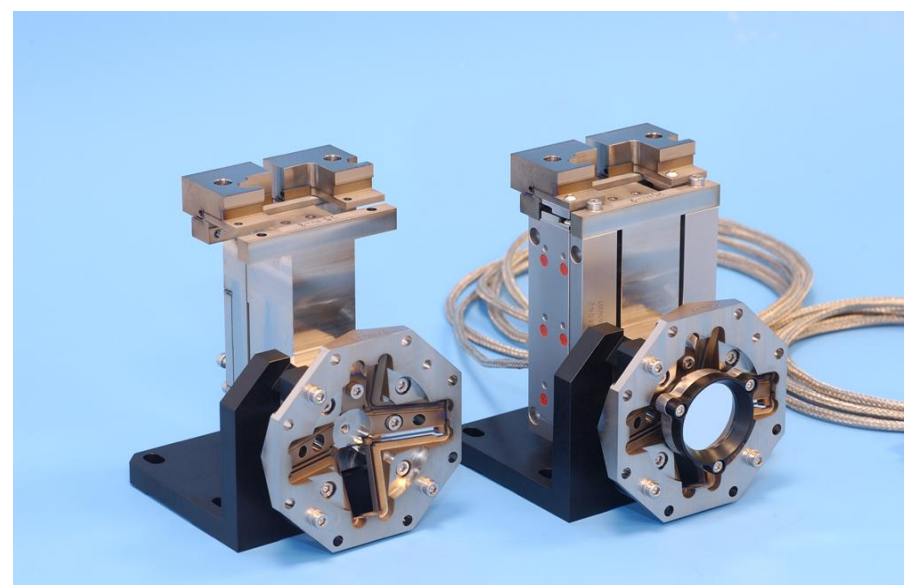

Figure 6: PRIMA AT Star Separator M11 mechanisms 


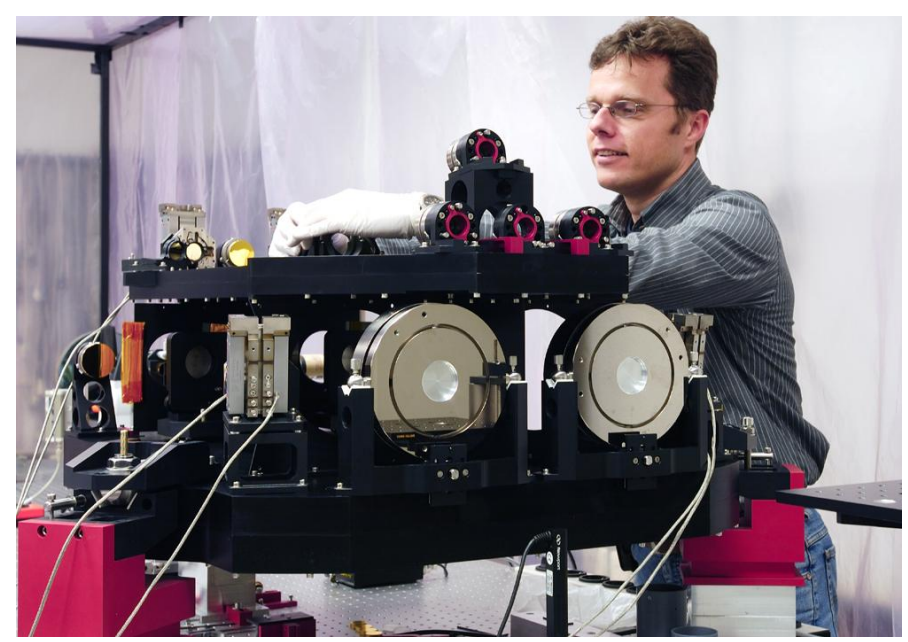

Figure 7: AT Star Separator testing at TNO

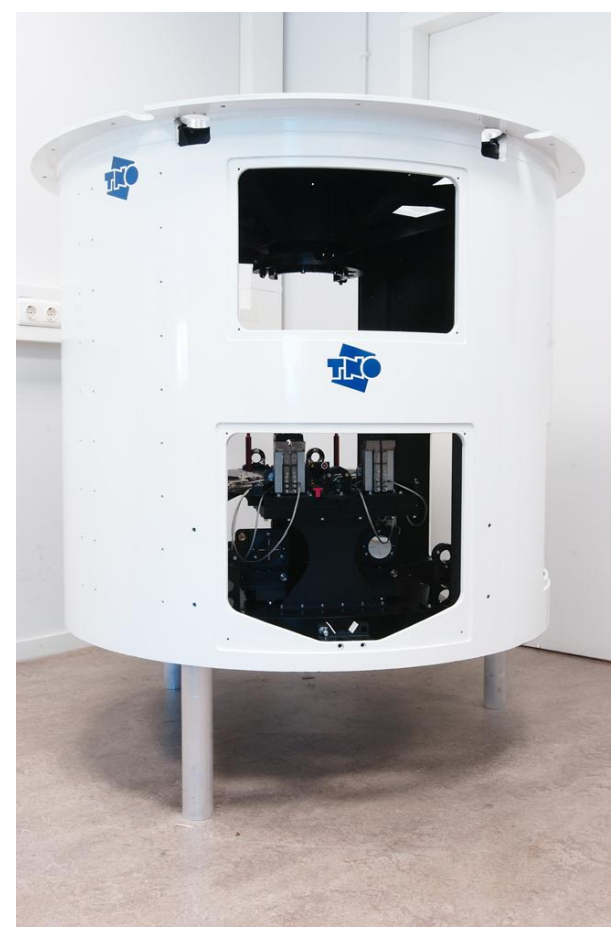

Figure 8: AT Star Separator Relay Optics Structure (ROS)

\section{UT STAR SEPARATOR}

De UT Star Separator works along the same principle as the AT Star Separator, but has its own de-rotator mechanism (on M10) and a scan mechanism with extended travel, called Field Selector (FS).

The function of the FS mechanism is to select any 2" sub-field out of a field of view of 120". Two of these mechanisms enable the selection of the guide star and the science object. 
The M10 mechanism enables the actual star separation. Due to the earth rotation the image of the hemisphere in the Coude focal plane rotates as function of time. Therefore the M10 mechanism is also equipped with a function to follow the rotating star image.

\subsection{FS mechanism}

The Field selector consists of a guiding mechanism and two actuators (Figure 9). To avoid friction effects it would be best to make the whole mechanism as a monolithic structure with flexures. For practical reasons this is not always possible meaning that the mechanism has to be split in several components. By connecting them with push/pull connections, friction effects are avoided. FS mechanism has been split into a number of components being:

1. Monolithic guiding mechanism.

2. Push/pull struts (3x)

3. A monolithic actuator mechanism that provides the required gear ratio between the piezo $(38 \mu \mathrm{m}$ travel $)$ and the actuator strut translation of about $2 \mathrm{~mm}$

All parts are made from a Titanium alloy (Ti6Al4V) because this material provides the best elastic performance.

The actuator levers to which the struts are connected have been light weighted as much as possible. This is for dynamic reasons. The gear ratio obtained through these levers is 11 . The other levers provide a gear ratio of 5 giving a total gear ratio of 55. These upper levers have been split in two sections allowing to adjust the angular position of the mirror for a given piezo position. The piezo pushes against the lever. To make sure that contact between the piezo and the lever is never lost a small preload spring is added. The FS actuators are equipped with magnetic dampers to avoid internal resonances Figure 10. The assembled FS mechanisms are shown in Figure 11.
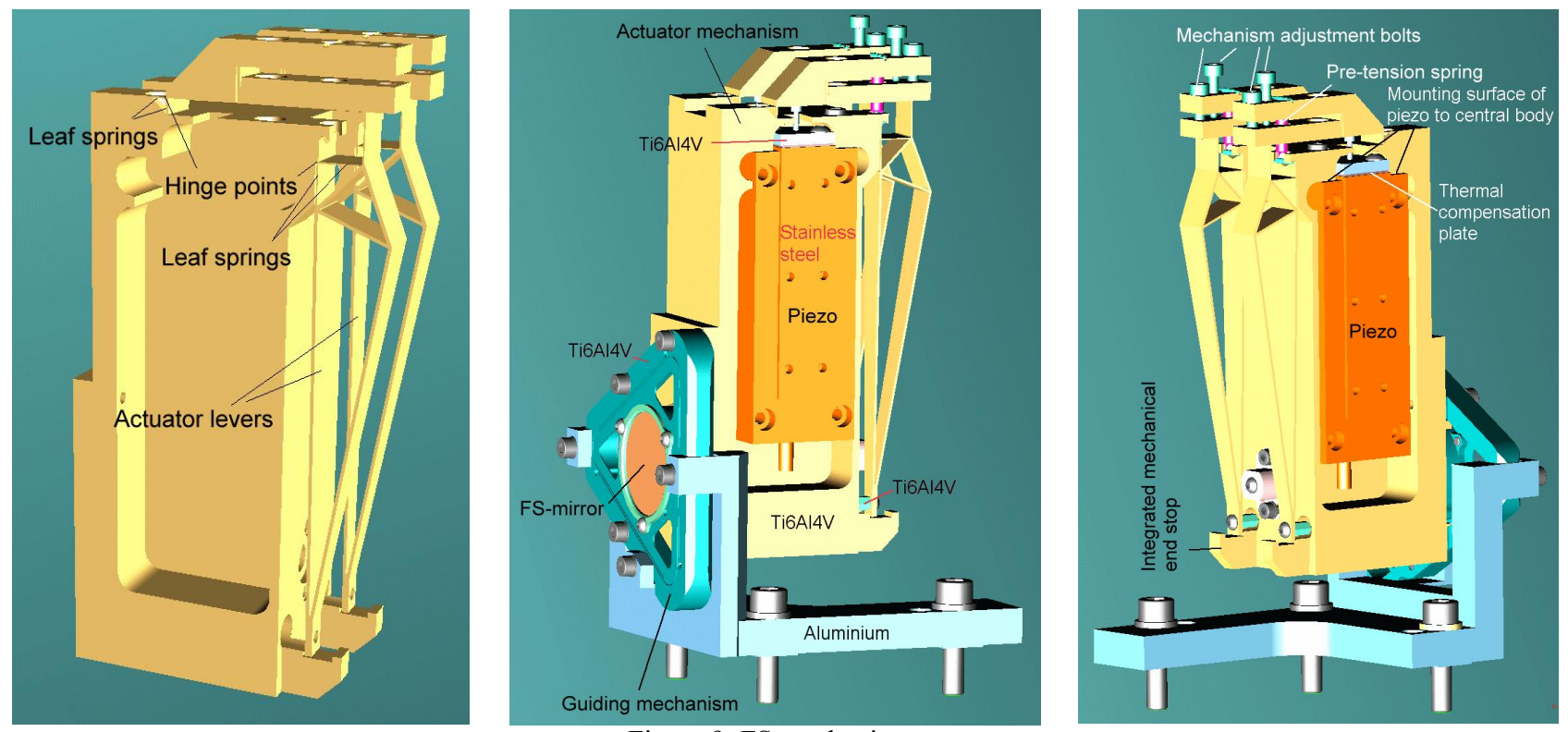

Figure 9: FS mechanism 


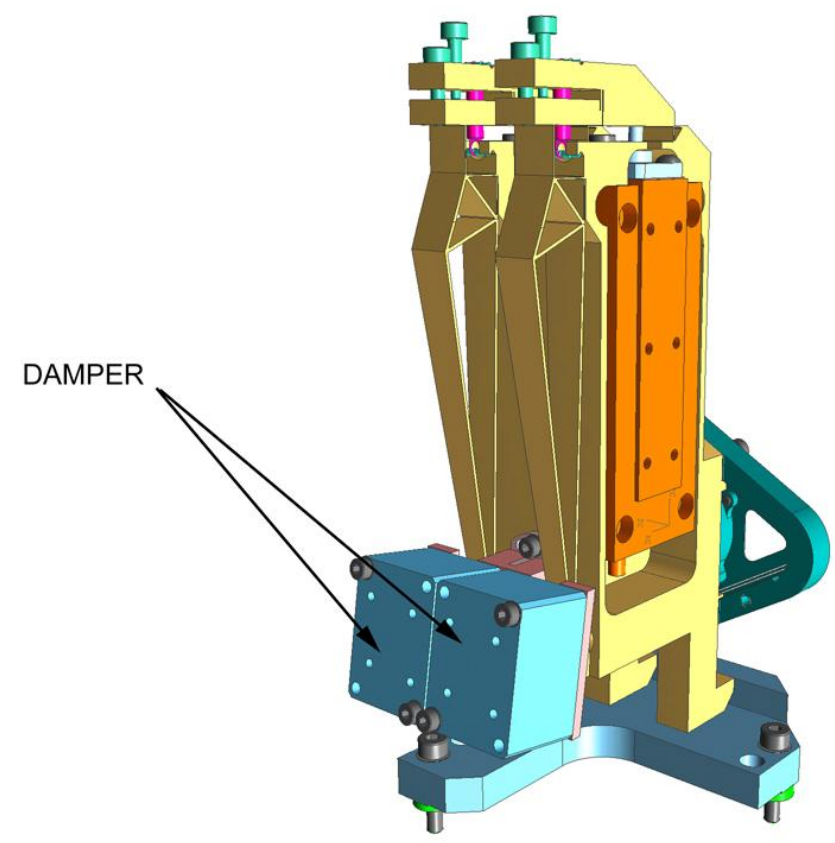

Figure 10: UT Star Separator scan mirrors with magnetic dampers

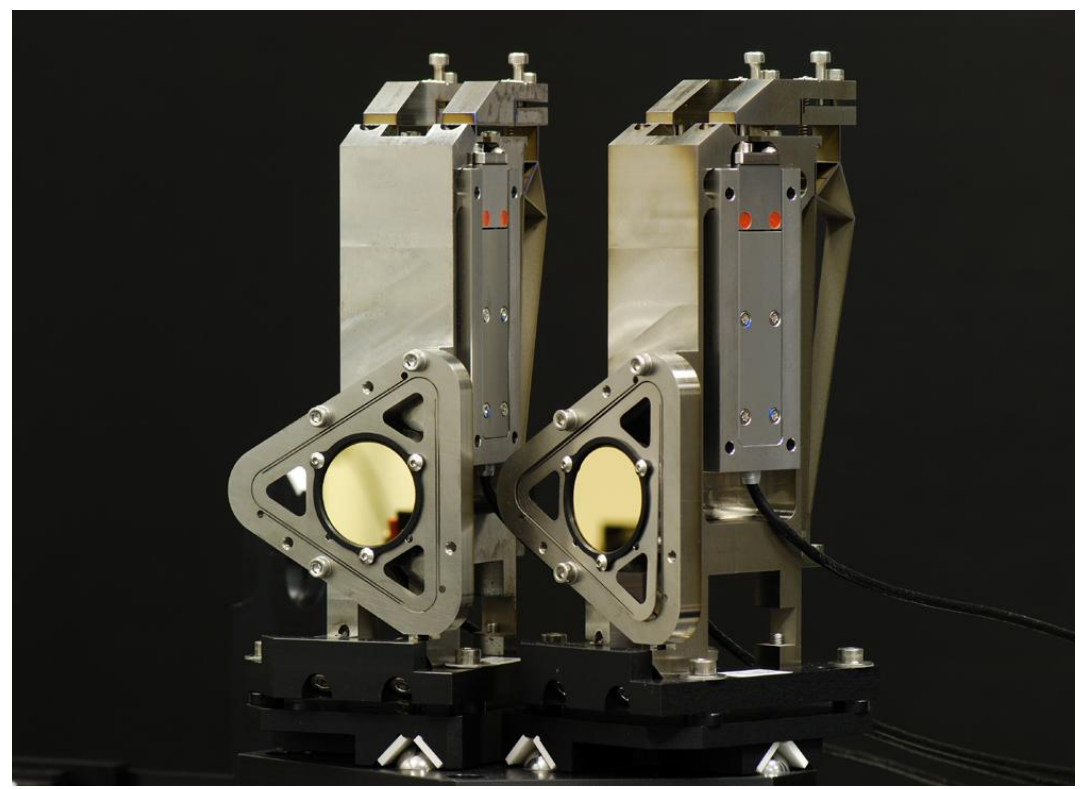

Figure 11: PRIMA UT Star Separator Field Selector mechanisms

The Field Selector mechanism is a much improved version of the mechanism of REF.2. That becomes visible when the most important performance parameters are compared.

Table 2: Comparison between mechanisms for AT and UT 


\begin{tabular}{|l|l|l|}
\hline Characteristic & STS-AT & STS-UT \\
\hline Mirror rotation angle & $16 \mathrm{mrad}$ & $129 \mathrm{mrad}$ \\
\hline Rotational stiffness in plane of mirror & & $90 \mathrm{x}$ smaller \\
\hline Thickness of struts & $\varnothing 0.8 \mathrm{~mm}$ & $\emptyset 0.4 \mathrm{~mm}$ \\
\hline Moment of inertia of mirror assembly & & $6 \mathrm{x}$ smaller \\
\hline Moving mass of actuator lever & $42 \mathrm{gr}$. & 7 gr. \\
\hline Stiffness of actuator lever & & $5 \times$ smaller \\
\hline
\end{tabular}

Further enhancements of the mechanism relative to the mechanism for the STS-AT are:

- Both rotation axes of the mirror coincides with the mirror surface hence no OPD effects.

- The actuation direction of the piezo is perpendicular now to the actuated lever.

- The pin that is mounted on top of the piezo has a much reduced lateral stiffness thereby increasing the threshold for potential friction effects at the contact point with the lever.

- The actuating direction of the actuator lever has reversed. Instead of pushing, the lever is pulling on the actuator struts.

- A tension spring provides a $5 \mathrm{~N}$ hysteresis free preload on the piezo.

- The adjustments of the mechanism are simplified.

The dynamic performance of the mechanisms has not changed. That means that chopping the mirror at $50 \mathrm{~Hz}$ is still possible.

\subsection{M10 pointing and tracking mechanism}

The principle of operation of the M10-mechanism is shown in Figure 12. Both M10 mirrors have to rotate around axes that are defined by the point of contact of the two mirrors and the centre of curvature of the mirror section. Rotating the mirror will not cause a change in optical configuration because the actual mirror surface will always be a subsection of the same sphere. Both mirror sections have to rotate at equal speed to avoid a collision between the mirrors. From the figure in can be concluded that rotating the mirrors will cause that the mirror surfaces will rotate relative to each other. This can be understood when one realises that the mirror assembly, from an optical point of view, consists of two mirrors that are tilted relative to each other whereby they have a common vertex. The mirrors will have a sharp edge to allow one star to be imaged by the telescope on the intersection of the two mirrors. The gap between the two mirrors will be about $20 \mu \mathrm{m}$. Depending on where the telescope re-images the stars on the mirrors a different mirror sub-set is used.

The rotation function is provided by a piezo motor. The advantage over a conventional gearbox is the lack of play and backlash. The de-rotator mechanism is shown in Figure 13. 


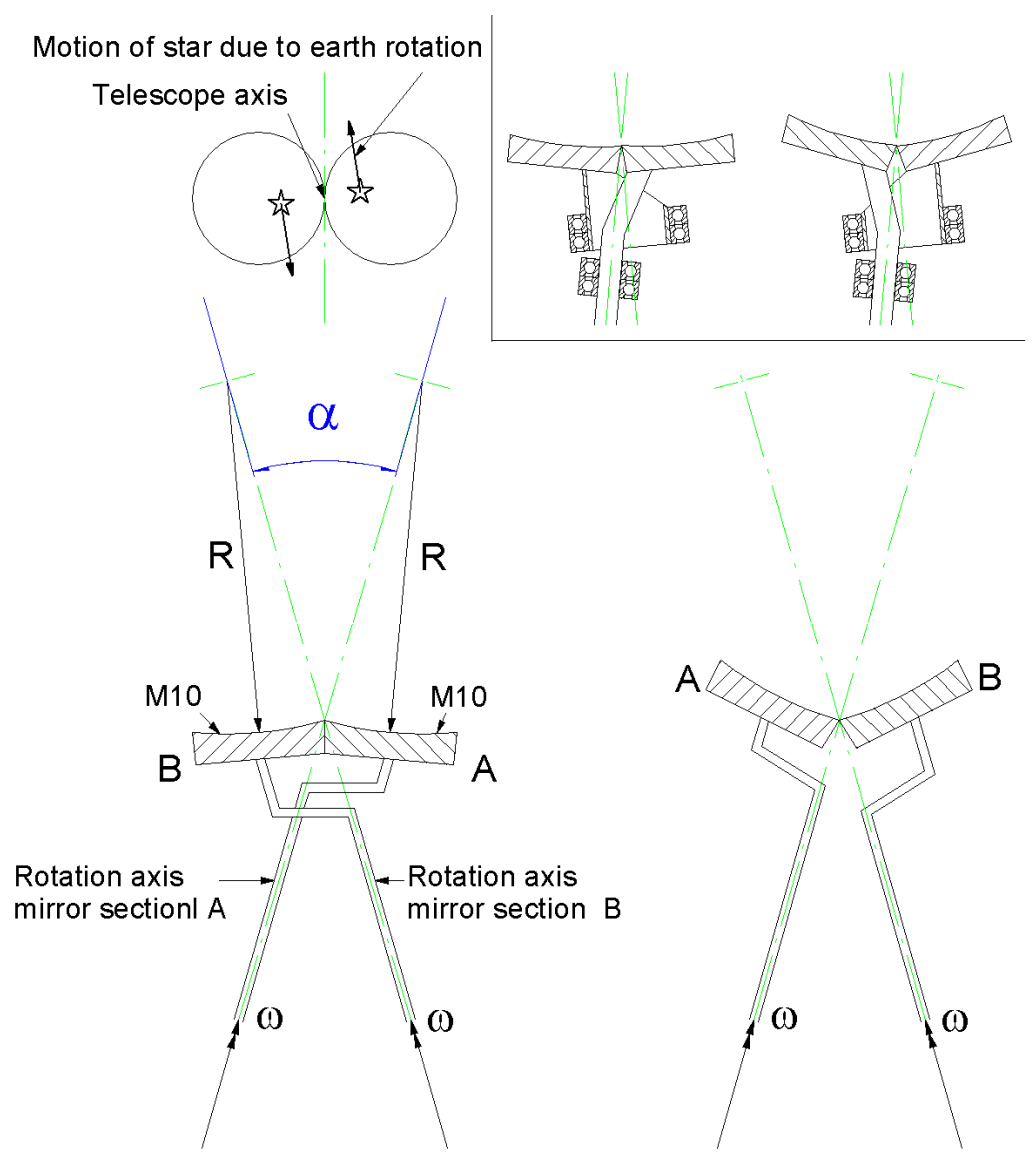

Note: Mirror surfaces coincide with Coude focal plane

Figure 12: Principle of operation of M10-mechanism

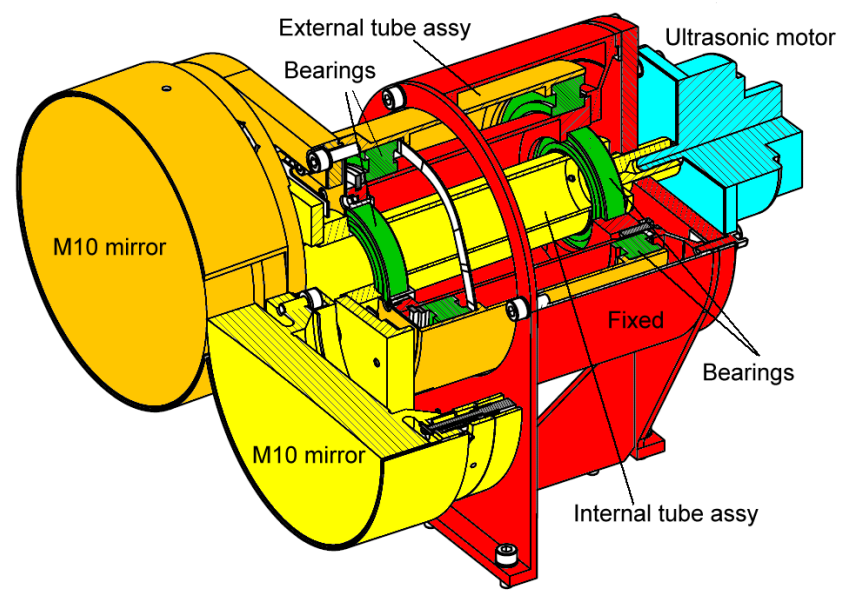

Figure 13: UT M10 pointing and tracking mechanism 
Other important features of the mechanism are:

- The mirrors are mounted on a kinematic mount which allows (dis)mounting without loosing the alignment of the mirrors.

- The gap between the two mirrors will be about $30 \mu \mathrm{m}$. About $10 \mu \mathrm{m}$ is used for the actual gap while the mirror edges have an area of around $10 \mu \mathrm{m}$ width that is slightly deviating from the exact mirror radius.

- The mirrors will have a sharp edge to allow one star to be imaged by the telescope on the intersection of the two mirrors.

- A protection ring has been fitted around the mirror for safety reasons. The mirror is razor sharp at the edge.

- The mirrors are mounted on a kinematic mount and held in position with bayonet type of pins. This is of particular importance for transport reasons. The mirror can now be packed separately without the need for realignment when the instrument is assembled at Paranal.

The fully assembled UT Star Separator is shown in Figure 14.

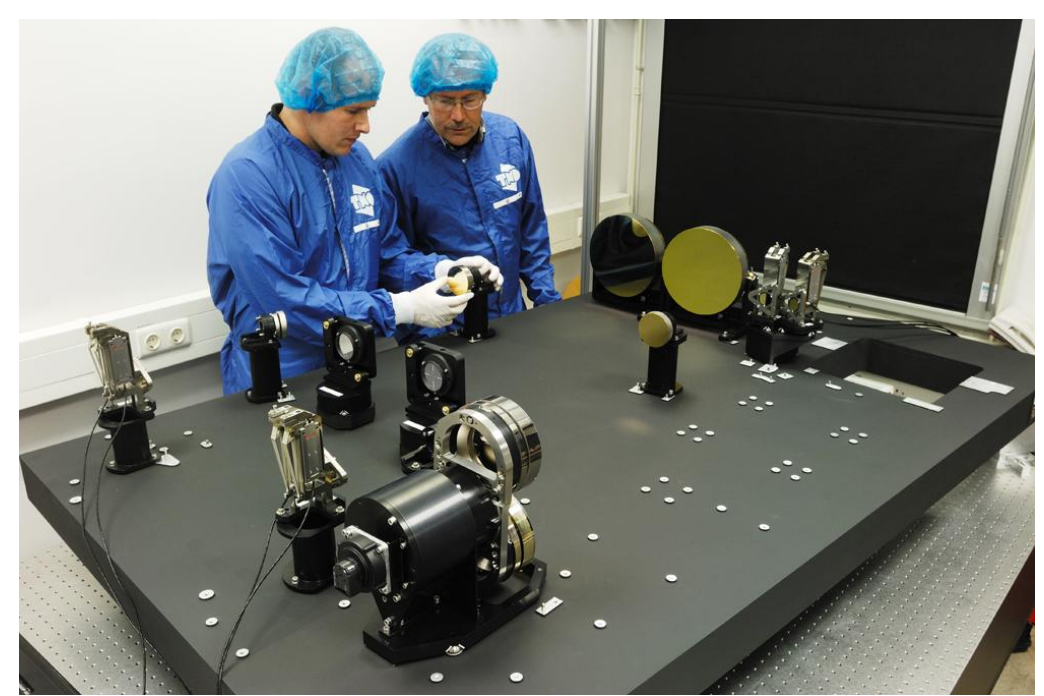

Figure 14: PRIMA UT Star Separator with M10 pointing and tracking mechanism in foreground

\section{CONCLUSIONS}

TNO has successfully developed the Star Separator sub systems for the AT and UT telescopes. The Star Separators meet or surpasses the requirements (Table 3).

It has been demonstrated that elastic mechanisms can operate very predictably and that there is no need for control feedback on such mechanism.

Reproducibility of mirror mounting has also been demonstrated, even after re-polishing a number of mirrors. Isostatic mirror mounts ensure precise and repeatable positioning at sub-micron level.

Table 3: Star Separator performance

\begin{tabular}{|l|l|l|}
\hline Requirement & Required value (on sky) & Obtained value (on sky) \\
\hline Pointing range & $120 "$ & $\begin{array}{l}149 \text { "(vertical) } \\
130 \text { ”(horizontal) }\end{array}$ \\
\hline Pointing accuracy & \pm 0.01 " & \pm 0.01 ” \\
\hline Pointing resolution & $\pm 0.002 "$ & $\pm 0.002 "$ \\
\hline Pointing correction freq. & $50 \mathrm{~Hz}$ & $100 \mathrm{~Hz}$ \\
\hline Chopping frequency & $1 \mathrm{~Hz}(5 \mathrm{~Hz}$ goal) & $63 \mathrm{~Hz}$ \\
\hline
\end{tabular}




\begin{tabular}{|l|l|l|}
\hline Chopping accuracy & $\pm 0.44 ”$ & $\pm 0.01 \%$ \\
\hline
\end{tabular}

The optical performance of the Star Separators is well within required value of $35 \mathrm{~nm}$ RMS. The measured Wave Front Error (WFE) of the Star Separators ranges from 25 - $30 \mathrm{~nm}$ RMS (Figure 15.

The sharp edge of the M10 mirrors gives a much better split of the optical beam as predicted with also much less stray light than expected.

OPD stability is around $9 \mathrm{~nm}$ RMS for all UT Star Separators and even less for the AT Star Separators.

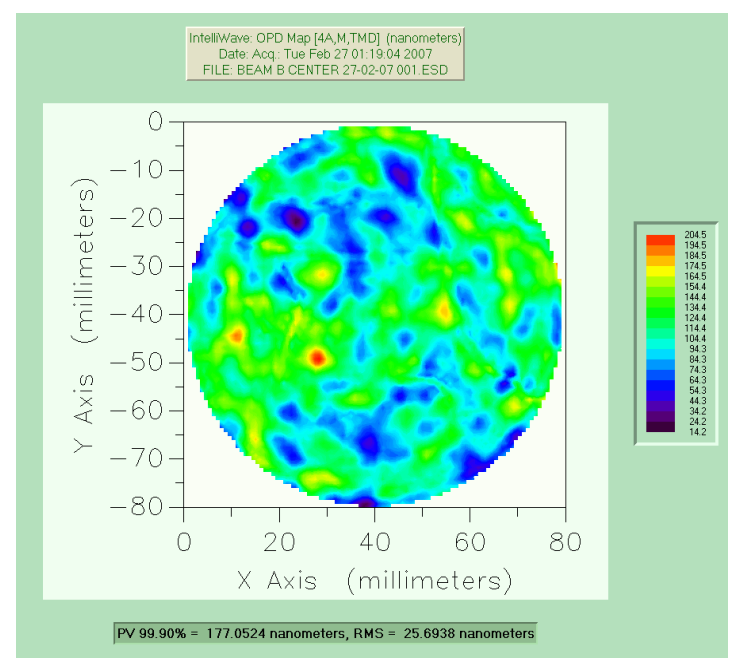

Figure 15: WFE of Beam B of AT Star Separator \#2

Two AT- and two UT-Star Separators have been delivered to ESO for integration in the VLTI PRIMA facility at Cerro Paranal (Figure 16). TNO is currently finalizing the assembly and acceptance testing of another two UT Star Separators. 


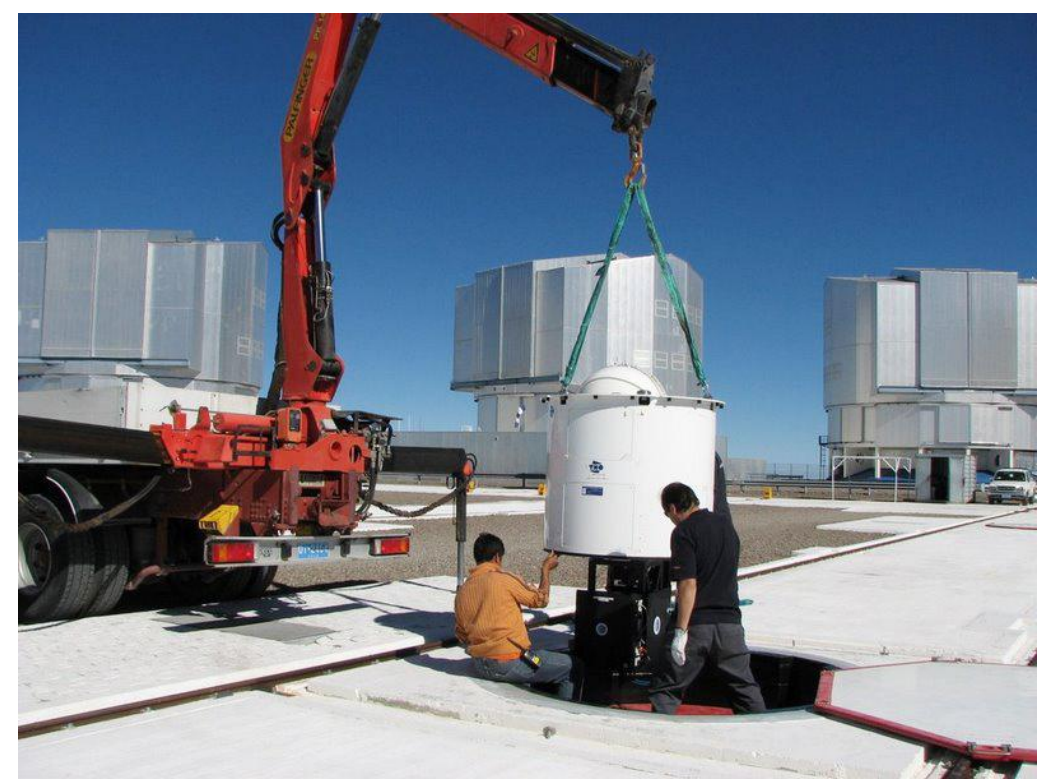

Figure 16: AT Star Separator ROS integration in one of the station pits in Paranal (courtesy Frederic Derie)

\section{ACKNOWLEDGEMENT}

The work reported in this paper was conducted at TNO under a contract with ESO in Garching (Germany). We would like to thank Frederic Derie and Francoise Delplancke at ESO, for their input during the design and development of the Star Separator subsystems. TNO also wishes to thank subcontractor Axsys for their excellent work on the Star Separator mirrors.

\section{REFERENCES}

1. F.Derie et al, "PRIMA Technical Description and Implementation", Workshop "Hunting for Planets ", Lorentz center, Leiden University, 3-6 June 2002.

2. F. Delplancke et al, "Star separator system for the dual-field capability (PRIMA) of the VLTI", SPIE New Frontiers in Stellar Interferometry, Vol. 5491, 2004

3. J. Nijenhuis et al, "Two axes fully elastic pointing mechanism at microarcsec resolution", SPIE Space Systems Engineering and Optical Alignment Mechanisms, Vol. 5528, 2004

4. J. Nijenhuis et al, "Mechanisms enabling observation of Jupiter like planets in deep space", SPIE Optomechanics, Vol. 5877, 2005

5. J. Nijenhuis et al, "A major step forward back in time with the ESO Star Separator system", SPIE Optomechanics, Vol. 5877, 2005

6. F. Delplancke et al, "PRIMA for the VLTI: a status report", SPIE Advances in Stellar Interferometry, Vol. 6268, 2006 